\title{
Corrección de riesgo relativo en sepsis neonatal
}

\author{
Carlos Martín Corral-Chávez
}

Universidad Autónoma de Durango, Campus Los Mochis, Escuela de Medicina, Sinaloa, México

Respetable editor,

Leí con interés el artículo "Exposición prolongada a antibióticos y riesgo de sepsis tardía (ST) en neonatos de 1000 a < 1500 g: estudio de cohorte", del doctor Briones Lara et al., ${ }^{1}$ publicado en Gaceta Médica de México.

Los autores calcularon el riesgo relativo (RR) en dos grupo de neonatos: expuestos (recibir antibióticos $>5$ días) y no expuestos (< 5 días), con la probabilidad de desarrollar sepsis tardía y enterocolitis necrosante en neonatos prematuros con peso corporal de 1000 a $<1500 \mathrm{~g}$. Reportaron una incidencia de sepsis tardía de $65.3 \%$ (32/49) en los expuestos y de $8.1 \%(4 / 49)$ en los no expuestos, y de enterocolitis necrosante de $24.5 \%(12 / 49)$ y $4.0 \%(2 / 49)$, respectivamente.

El RR calculado para desarrollar sepsis tardía indicado fue de 21.1 (IC $95 \%=6.5-68.9$ ), dato que en realidad corresponde a la razón de momios (RM); el RR corregido para sepsis tardía debe ser 8.6 (IC $95 \%=3.05-20.9$ ). De la misma forma, el RR para desarrollar enterocolitis necrosante indicado fue 7.6 (IC $95 \%=1.6-36.1$ ), que debe ser 6.0 (IC $95 \%=$ 1.4-25.4). La corrección del RR se realizó en Epi-info versión 7.2 para Window. ${ }^{2}$

En los estudios de cohorte podemos estimar el RR y la RM para medir la fuerza de asociación, pero existen condiciones para considerar que estas mediciones sean equivalentes; la más importante es que la enfermedad de interés presente una frecuencia baja en el grupo expuesto. ${ }^{3}$ Cuando se cumple esta condición, el RR y la RM son una buena aproximación y se pueden utilizar de manera indistinta. En el reporte comentado, la incidencia de los desenlaces fue muy alta, $65.3 \%$ (32/49) desarrolló sepsis tardía y $24.5 \%$ (12/49), enterocolitis necrosante. Por la elevada frecuencia en los expuestos, el RR y la RM no debieron utilizarse de manera indistinta.

El RR corregido para sepsis tardía y enterocolitis necrosante muestra una fuerte asociación entre el tiempo de exposición a antibióticos y el desarrollo de desenlaces desfavorables (sepsis tardía y enterocolitis necrosante).

Las observaciones anteriores de ninguna forma tienen la intención de invalidar los resultados reportados en el estudio.

\section{Bibliografía}

1. Briones-Lara E, Treviño-Báez J, Caballero-Trejo A, Irruegas-Maeda A, Palacios-Saucedo GC, Ramírez-Rosalino MC. Exposición prolongada a antibióticos y riesgo de sepsis tardía (ST) en neonatos de 1,000 a $<$ 1,500 g: estudio de cohorte. Gac Med Mex 2015;151:306-312.

2. Epi-info for Window V7.2. Disponible en: https://www.cdc.gov/epiinfo/ support/downloads.html.

3. Gordis L. Epidemiología. España: Elsevier-Saunders; 2005. 